\title{
THE TENSOR PRODUCT PROBLEM FOR REFLEXIVE ALGEBRAS
}

\author{
JON KRAUS
}

It was observed by Gilfeather, Hopenwasser, and Larson in [1] that Tomita's commutation formula for tensor products of von Neumann algebras can be rewritten in a way that makes sense for tensor products of arbitrary reflexive algebras. The tensor product problem for reflexive algebras is to decide for which pairs of reflexive algebras this tensor product formula is valid.

Recall that a subalgebra $\mathscr{M}$ of the algebra $B(\mathscr{H})$ of all bounded operators on a Hilbert space $\mathscr{H}$ is said to be a von Neumann algebra if it is closed in the weak operator topology, contains the identity operator $I$, and is self-adjoint (i.e., $A \in \mathscr{M}$ implies $A^{*} \in$ $\mathscr{M})$. The commutant $\mathscr{M}^{\prime}$ of $\mathscr{M}$ is the set of all operators $B \in$ $B(\mathscr{H})$ such that $B A=A B$ for all $A \in \mathscr{M}$. The commutant of a von Neumann algebra is again a von Neumann algebra. Moreover, it follows from von Neumann's double commutant theorem that a self-adjoint subalgebra $\mathscr{M}$ of $B(\mathscr{H})$ is a von Neumann algebra if and only if $\mathscr{M}=\mathscr{M}^{\prime \prime}$.

Let $\mathscr{M} \subset B(\mathscr{H})$ and $\mathscr{N} \subset B(\mathscr{K})$ be von Neumann algebras, and let $\mathscr{H} \otimes \mathscr{K}$ denote the Hilbert space tensor product of $\mathscr{H}$ and $\mathscr{K}$. If $A \in \mathscr{M}$ and $B \in \mathscr{N}$, there is a unique operator $A \otimes B$ in $B(\mathscr{H} \otimes \mathscr{K})$ such that $(A \otimes B)(x \otimes y)=A x \otimes B y$ for all $x \in \mathscr{H}$ and $y \in \mathscr{K}$. The von Neumann algebra generated by $\{A \otimes B \mid A \in \mathscr{M}$ and $B \in \mathscr{N}\}$ is denoted by $\mathscr{M} \bar{\otimes} \mathscr{N}$. Tomita's commutation theorem asserts that for any pair of von Neumann algebras $\mathscr{M}$ and $\mathscr{N}$ the following commutation formula is valid:

$$
\mathscr{M}^{\prime} \bar{\otimes} \mathscr{N}^{\prime}=(\mathscr{M} \bar{\otimes} \mathscr{N})^{\prime} .
$$

A number of results concerning tensor products of von Neumann algebras follow from Tomita's theorem. (See, for example, §IV.5 of [13].) 1989.

Received by the editors August 23, 1989 and, in revised form, December 8,

1980 Mathematics Subject Classification (1985 Revision). Primary 47D25, $46 \mathrm{~L} 10$.

Research partially supported by grants from the National Science Foundation. 
A sublattice of the lattice of (self-adjoint) projections in $B(\mathscr{C})$ is said to be a subspace lattice if it is strongly closed and contains 0 and $I$. If $\mathscr{L}$ is a subspace lattice, we let

$$
\text { alg } \mathscr{L}=\{A \in B(\mathscr{H}) \mid P A P=A P \text { for all } P \in \mathscr{L}\} .
$$

Note that alg $\mathscr{L}$ is a unital $\sigma$-weakly closed subalgebra of $B(\mathscr{H})$. If $\mathscr{A}$ is a subalgebra of $B(\mathscr{H})$, we let lat $\mathscr{A}$ denote the subspace lattice consisting of all the projections that are left invariant by every element of $\mathscr{A}$. An algebra $\mathscr{A}$ is reflexive if $\mathscr{A}=$ alg lat $\mathscr{A}$. It is easy to see that the reflexive algebras are precisely the algebras of the form alg $\mathscr{L}$ for some subspace lattice $\mathscr{L}$, and that the selfadjoint reflexive algebras are precisely the von Neumann algebras. Moreover, if we let $\mathscr{L}_{1}$ and $\mathscr{L}_{2}$ be the projection lattices of the von Neumann algebras $\mathscr{M}$ and $\mathscr{N}$, respectively, then (1) becomes

$$
\operatorname{alg} \mathscr{L}_{1} \otimes \operatorname{alg} \mathscr{L}_{2}=\operatorname{alg}\left(\mathscr{L}_{1} \otimes \mathscr{L}_{2}\right),
$$

where $\mathscr{L}_{1} \otimes \mathscr{L}_{2}$ is the subspace lattice generated by $\left\{P_{1} \otimes P_{2} \mid P_{i} \in\right.$ $\left.\mathscr{L}_{i}\right\}$.

Equation (2) makes sense for any pair of reflexive algebras alg $\mathscr{L}_{1}$ and alg $\mathscr{L}_{2}$, and the tensor product problem for reflexive algebras is: for which pairs of reflexive algebras is equation (2) valid? As noted above, this problem was first raised in [1], where it was shown that (2) is valid when both subspace lattices are nests (totally ordered). It has since been shown that (2) is valid for large classes of reflexive algebras [3-8]. However, it has remained an open question whether (2) is always valid. The main purpose of this note is to announce that the answer to this question is no. Perhaps surprisingly, (2) can fail even when one of the reflexive algebras is a von Neumann algebra. In fact, we can prove the following result.

Theorem 1. Let $\mathscr{H}$ be a separable infinite-dimensional Hilbert space. Then for each of the types $\mathrm{II}_{1}, \mathrm{II}_{\infty}$, and $\mathrm{III}_{\lambda}(0 \leq \lambda \leq 1)$, there is a factor $\mathscr{M}$ of that type acting on $\mathscr{H}$ and a reflexive algebra $\mathscr{A} \subset B(\mathscr{H})$ such that if alg $\mathscr{L}_{1}=\mathscr{M}$ and alg $\mathscr{L}_{2}=\mathscr{A}$, then $\operatorname{alg} \mathscr{L}_{1} \otimes \operatorname{alg} \mathscr{L}_{2} \neq \operatorname{alg}\left(\mathscr{L}_{1} \otimes \mathscr{L}_{2}\right)$.

The proof of Theorem 1 and the other results announced in this note will appear in [9]. The proof of Theorem 1 depends on a reformulation of Tomita's commutation theorem in terms of slice maps, due to Tomiyama [14]. If $\mathscr{H}$ and $\mathscr{K}$ are Hilbert spaces, and $\varphi$ is in the predual $B(\mathscr{H})_{*}$ of $B(\mathscr{H})$, the right slice map 
$R_{2}$ is the unique $\sigma$-weakly continuous map from $B(\mathscr{H}) \bar{\otimes} B(\mathscr{K})$ to $B(\mathscr{K})$ such that $R_{2}(A \otimes B)=\varphi(A) B$ for all $A \in B(\mathscr{H})$ and $B \in B(\mathscr{K})$. The left slice maps $L_{\psi}$ are similarly defined. If $\mathscr{S} \subset$ $B(\mathscr{H})$ and $\mathscr{T} \subset B(\mathscr{K})$ are $\sigma$-weakly closed (linear) subspaces, then $\mathscr{S} \bar{\otimes} \mathscr{T}$ is the $\sigma$-weakly closed linear span of $\{S \otimes T \mid S \in \mathscr{S}$ and $T \in \mathscr{T}\}$. The Fubini product $F(\mathscr{S}, \mathscr{T})$ of $\mathscr{S}$ and $\mathscr{T}$ is the $\sigma$-weakly closed subspace of $B(\mathscr{H}) \bar{\otimes} B(\mathscr{K})$ consisting of those operators $A$ all of whose right slices $R_{2}(A)$ are in $\mathscr{T}$ and all of whose left slices $L_{\psi}(A)$ are in $\mathscr{S}$. It is immediate that $\mathscr{S} \bar{\otimes} \mathscr{T} \subset F(\mathscr{S}, \mathscr{T})$.

Tomiyama showed in [14] that Tomita's theorem is equivalent to the statement that $F(\mathscr{M}, \mathscr{N})=\mathscr{M} \bar{\otimes} \mathscr{N}$ whenever $\mathscr{M}$ and $\mathscr{N}$ are von Neumann algebras. The slice map problem is: find all pairs of ( $\sigma$-weakly closed) subspaces $\mathscr{S}$ and $\mathscr{T}$ for which

$$
F(\mathscr{S}, \mathscr{T})=\mathscr{S} \bar{\otimes} \mathscr{T} \text {. }
$$

As we observed in [6],

$$
F\left(\operatorname{alg} \mathscr{L}_{1}, \operatorname{alg} \mathscr{L}_{2}\right)=\operatorname{alg}\left(\mathscr{L}_{1} \otimes \mathscr{L}_{2}\right)
$$

for any pair of reflexive algebras alg $\mathscr{L}_{1}$ and alg $\mathscr{L}_{2}$, so (2) is valid if and only if (3) holds with $\mathscr{S}=\operatorname{alg} \mathscr{L}_{1}$ and $\mathscr{T}=\operatorname{alg} \mathscr{L}_{2}$.

A subspace $\mathscr{S}$ of $B(\mathscr{H})$ is said to have Property $S_{\sigma}$ [6] if for every Hilbert space $\mathscr{K}$ we have that $F(\mathscr{S}, \mathscr{T})=\mathscr{S} \bar{\otimes} \mathscr{T}$ for every subspace $\mathscr{T}$ of $B(\mathscr{K})$. It is immediate from (4) that if alg $\mathscr{L}_{1}$ has Property $S_{\sigma}$, then (2) is valid for alg $\mathscr{L}_{1}$ and any alg $\mathscr{L}_{2}$. We showed in [8] that the converse is true. Moreover, using various stability properties of the class of von Neumann algebras with Property $S_{\sigma}$, we can show that if for any of the types $\mathrm{II}_{1}, \mathrm{II}_{\infty}$, and $\mathrm{III}_{\lambda}(0 \leq \lambda \leq 1)$ all separably acting factors of that type have Property $S_{\sigma}$, then every von Neumann algebra has Property $S_{\sigma}$. Hence to prove Theorem 1 it suffices to show that there is a von Neumann algebra without Property $S_{\sigma}$. The proof of the existence of such a von Neumann algebra depends on a characterization of Property $S_{\sigma}$ in terms of an approximation property which we will now describe.

First, some notation. If $\mathscr{S}$ is a subspace of $B(\mathscr{H})$, and $\Phi$ is a bounded (linear) map from $\mathscr{S}$ to $\mathscr{S}$, then for each positive integer $n$ we let $\Phi_{n}$ denote the map from $\mathscr{S} \bar{\otimes} M_{n}(\mathbf{C})$ to itself defined by $\Phi_{n}\left(\left[S_{i j}\right]\right)=\left[\Phi\left(S_{i j}\right)\right]$ (where we make the usual identification of $\mathscr{S} \bar{\otimes} M_{n}(\mathrm{C})$ with $\left.M_{n}(\mathscr{S})\right)$. We say that $\Phi$ is completely bounded if $\|\Phi\|_{c b}=\sup \left\{\left\|\Phi_{n}\right\|: n \in \mathbf{N}\right\}$ is finite (cf. [11]). Let $\mathscr{K}$ 
be a fixed Hilbert space, and suppose $\Phi$ is a $\sigma$-weakly continuous completely bounded map from $\mathscr{S}$ to $\mathscr{S}$. Then there is a (unique) $\sigma$-weakly continuous map $\widetilde{\Phi}$ from $S \bar{\otimes}(\mathscr{K})$ to itself such that

$$
\widetilde{\Phi}(S \otimes T)=\Phi(S) \otimes T \quad(S \in \mathscr{S}, T \in B(\mathscr{K})) .
$$

Moreover, $\|\widetilde{\Phi}\|=\|\Phi\|_{c b}$.

We let $F(\mathscr{S})$ denote the collection of all $\sigma$-weakly continuous finite rank maps from $\mathscr{S}$ to $\mathscr{S}$, and for $A \in \mathscr{S} \bar{\otimes} B(\mathscr{K})$, we let $F(A)$ denote the $\sigma$-weak closure of $\{\widetilde{\Phi}(A) \mid \Phi \in F(\mathscr{S})\}$. (Note that a bounded finite rank map is automatically completely bounded.) We say that $\mathscr{S}$ has the (weak ${ }^{*}$-) complete pointwise approximation property (CPWAP) if there is a net $\left\{\Phi_{\alpha}\right\}$ in $F(\mathscr{S})$ such that $\widetilde{\Phi}_{\alpha}(A) \rightarrow A \sigma$-weakly for every $A \in \mathscr{S} \bar{\otimes}(\mathscr{K})$. (This definition appears to depend on $\mathscr{K}$, but it can be shown that if such a net exists for some separable infinite-dimensional Hilbert space, then such a net exists for any Hilbert space.) A subspace $\mathscr{S}$ has the $\left(\right.$ weak $^{*}$-) completely bounded approximation property (CBAP) [2] if there is a net $\left\{\Phi_{\alpha}\right\}$ in $F(\mathscr{S})$ such that $\sup \left\|\Phi_{\alpha}\right\|_{c b}<\infty$ and such that $\Phi_{\alpha}(S) \rightarrow S \quad \sigma$-weakly for all $S \in \mathscr{S}$. It is not hard to show that the CBAP implies the CPWAP. However, it follows from results in [2] that if $\Gamma$ is the semidirect product of $\mathbf{Z}^{2}$ by $S L(2, \mathbf{Z})$ under the natural action, then the group von Neumann algebra $\mathscr{M}(\Gamma)$ has the CPWAP but does not have the CBAP. It follows from our next result that $\mathscr{M}(\Gamma)$ has Property $S_{\sigma}$.

Theorem 2. Let $\mathscr{S}$ be a subspace of $B(\mathscr{H})$, and let $\mathscr{K}$ be an infinite-dimensional Hilbert space. Then the following are equivalent:

(a) $\mathscr{S}$ has Property $S_{\sigma}$.

(b) $A \in F(A)$ for every $A \in \mathscr{S} \bar{\otimes} B(\mathscr{K})$.

(c) $\mathscr{S}$ has the CPWAP.

The key step in the proof of Theorem 2 is the following proposition.

Proposition. Let $\mathscr{S}$ be a subspace of $B(\mathscr{H})$, and let $\mathscr{K}$ be a Hilbert space. Let $A \in \mathscr{S} \bar{\otimes}(\mathscr{K})$, and let $\mathscr{T}$ denote the $\sigma$-weak closure of $\left\{R_{2}(A) \mid \varphi \in B(\mathscr{H})_{*}\right\}$. Then $F(\mathscr{S}, \mathscr{T})=F(A)$. 
The next result, which plays an essential role in the proof of Theorem 1, is due to Uffe Haagerup, and we are grateful to Professor Haagerup for allowing us to include this result in [9].

Theorem 3 (Haagerup). If $\mathscr{S}$ has Property $S_{\sigma}$, then the predual $\mathscr{S}_{*}$ of $\mathscr{S}$ has the approximation property.

Recall that a Banach space $X$ is said to have the approximation property (AP) if for every compact subset $K$ of $X$ and every $\varepsilon>0$ there is a finite rank bounded linear map $T$ from $X$ to $X$ such that $\|T x-x\|<\varepsilon$ for all $x \in K$. There are a number of conditions on $X$ that are equivalent to the AP. The proof of Theorem 3 consists of showing that if $\mathscr{S}$ has the CPWAP, then $\mathscr{S}_{*}$ satisfies one of these conditions (condition (iv) of Theorem 1.e.4 in [10]).

In [12], Szankowski proved the remarkable result that $B(\mathscr{H})$ does not have the AP if $\mathscr{H}$ is infinite dimensional. Moreover, it is a standard fact that if $X^{*}$ has the AP, then so does $X$. Hence the von Neumann algebra $B(\mathscr{H})^{* *}$ does not have Property $S_{\sigma}$. As noted above, this implies Theorem 1.

\section{REFERENCES}

1. F. Gilfeather, A. Hopenwasser and D. Larson, Reflexive algebras with finite width lattices: tensor products, cohomology, compact perturbations, J. Funct. Anal. 55 (1984), 176-199.

2. U. Haagerup, Group $C^{*}$-algebras without the completely bounded approximation property, preprint.

3. A. Hopenwasser and J. Kraus, Tensor products of reflexive algebras II, J. London Math. Soc. (2) 28 (1983), 359-362.

4. A. Hopenwasser, C. Laurie and R. Moore, Reflexive algebras with completely distributive subspace lattices, J. Operator Theory 11 (1984), 91-108.

5. J. Kraus, $W^{*}$-dynamical systems and reflexive operator algebras, J. Operator Theory 8 (1982), 181-194.

6. _ The slice map problem for $\sigma$-weakly closed subspaces of von Neumann algebras, Trans. Amer. Math. Soc. 279 (1983), 357-376.

7. _ Tensor products of reflexive algebras, J. London Math. Soc. (2) 28 (1983), 350-358.

8. __ Abelian operator algebras and tensor products, J. Operator Theory 14 (1985), 391-407.

9. __ The slice map problem and approximation properties, preprint.

10. J. Lindenstrauss and L. Tzafiri, Classical Banach spaces I, Ergebnisse der Mathematik, no. 92, Springer-Verlag, New York, 1977.

11. V. I. Paulsen, Completely bounded maps and dilations, Pitman Research Notes in Math., no. 146, Longman Scientific \& Technical, New York, 1986. 
12. A. Szankowski, $B(\mathscr{H})$ does not have the approximation property, Acta Math. 147 (1981), 89-108.

13. M. Takesaki, Theory of operator algebras I, Springer-Verlag, New York, 1979.

14. J. Tomiyama, Tensor products and projections of norm one in von Neumann algebras, Lecture Notes, University of Copenhagen, 1970.

Department of Mathematics, State University of New York at Buffalo, Buffalo, New York 14214 